\title{
A combination of oral uracil-tegafur plus leucovorin (UFT + LV) is a safe regimen for adjuvant chemotherapy after hepatectomy in patients with colorectal cancer: Safety report of the UFT/LV study
}

\author{
Akio Saiura, ${ }^{1, *}$, Junji Yamamoto ${ }^{2}$, Kiyoshi Hasegawa ${ }^{3}$, Masaru Oba ${ }^{3}$, Tadatoshi Takayama ${ }^{4}$, \\ Shinichi Miyagawa ${ }^{5}$, Masayoshi Ijichi ${ }^{6}$, Masanori Teruya ${ }^{7}$, Fuyo Yoshimi ${ }^{8}$, Seiji Kawasaki ${ }^{9}$, \\ Hiroto Koyama $^{10}$, Masatoshi Makuuchi ${ }^{11}$, Norihiro Kokudo ${ }^{3}$ \\ ${ }^{1}$ Department of Gastrointestinal Surgery, Cancer Institute Hospital, Japanese Foundation for Cancer Research, Ariake Hospital, \\ Tokyo, Japan; \\ ${ }^{2}$ Department of Surgery, National Defense Medical College, Saitama, Japan; \\ ${ }^{3}$ Hepato-Biliary-Pancreatic Surgery Division, Department of Surgery, Graduate School of Medicine, The University of Tokyo, \\ Tokyo, Japan; \\ ${ }^{4}$ Department of Digestive Surgery, Nihon University School of Medicine, Tokyo, Japan; \\ ${ }^{5}$ First Department of Surgery, Shinshu University School of Medicine, Tokyo, Japan; \\ ${ }^{6}$ Department of Surgery, Social Insurance Chuo General Hospital, Tokyo, Japan; \\ ${ }^{7}$ Department of Surgery, Showa General Hospital, Tokyo, Japan; \\ ${ }^{8}$ Department of Surgery, Ibaraki Prefectural Central Hospital and Cancer Center, Ibaraki, Japan; \\ ${ }^{9}$ Department of Hepatobiliary-Pancreatic Surgery, Juntendo University School of Medicine, Tokyo, Japan; \\ ${ }^{10}$ Department of Surgery, Sempos Tokyo Takanawa Hospital, Tokyo, Japan; \\ ${ }^{11}$ Hepato-Biliary-Pancreatic Surgery and Transplantation Devision, Japanese Red Cross Medical Center, Tokyo, Japan.
}

Summary The use of adjuvant systemic chemotherapy for resectable liver metastases from colorectal cancer (CRC) is controversial because no trial demonstrated its benefit. We conducted the phase III trial to evaluate UFT/leucovorin ( $\mathrm{LV}$ ) for colorectal liver metastases (CRLM). The primary endpoint has not been available until 2014, we first report the feasibility and safety data of UFT/LV arm. In this multicenter trial, patients who underwent curative resection of liver metastases from colorectal cancer were randomly assigned to receive surgery alone or surgery followed by adjuvant chemotherapy with UFT/LV. The primary endpoint was relapse-free survival. Secondary endpoints included overall survival and safety. A total of 180 patients were enrolled, 90 were randomly assigned to receive UFT/LV therapy. Eighty two of whom were included in safety analyses. In the UFT/LV group, the completion rate of UFT/LV was $54.9 \%$, the relative dose intensity was $70.8 \%$ and grade 3 or higher adverse events occurred in $\mathbf{1 2 . 2 \%}$ of the patients. Elevated bilirubin levels, decreased hemoglobin levels, elevated alanine aminotransferase levels, diarrhea, anorexia were common. Most other adverse events were grade 2 or lower and tolerable. In conclusions, UFT/LV is a safe regimen for postoperative adjuvant chemotherapy in patients who have undergone resection of liver metastases from colorectal cancer. Further studies are warranted to improve completion rate, but $\mathrm{UFT} / \mathrm{LV}$ is found to be a promising treatment in this setting.

Keywords: Adjuvant treatment, colorectal cancer, randomized controlled trial, resection of liver metastases, UFT/LV

*Address correspondence to:

Dr. Akio Saiura, Department of Gastrointestinal Surgery, Cancer Institute Hospital, Japanese Foundation for Cancer Research, Ariake Hospital, 3-8-31 Ariake, Koto-ku, Tokyo 135-8550, Japan.

E-mail: saiura-tky@umin.ac.jp

\section{Introduction}

Hepatectomy is widely acknowledged to be therapeutically useful in patients with liver metastases from colorectal cancer (CRC), with a resection rate of $10 \%$ to $40 \%$ and a 5 -year survival rate of $30 \%$ to $45 \%$ 
(1-5). The aggressive extension of surgical indications has led to long-term survival even in patients with unfavorable prognostic factors $(6,7)$. However, relapse is common and occurs in approximately $75 \%$ of the patients (8).

Kokudo and his colleagues retrospectively analyzed 132 patients who had liver resection for colorectal metastasis at their hospital, they showed that adjuvant chemotherapy significantly improved surgical and disease-free survival after hepatic resection for colorectal metastases (9). Postoperative adjuvant chemotherapy is considered useful for inhibiting recurrence in the residual liver and the development of micrometastasis in patients who undergo resection of liver metastases. Several phase III clinical trials have previously compared surgery alone with surgery plus postoperative adjuvant chemotherapy, but clear-cut evidence demonstrating the effectiveness of postoperative adjuvant chemotherapy has yet to be obtained. This is reflected in the 2010 guidelines for the management of colorectal cancer issued by the Japanese Society for Cancer of Colon and Rectum. Despite this situation, FOLFOX (oxaliplatin, 5-fluorouracil, and leucovorin (LV)) therapy, which was shown to be effective for stage III and unresectable stage IV CRC (1014 ), has been widely used in routine medical practice. However, the feasibility and safety of postoperative FOLFOX therapy in patients undergoing hepatectomy has yet to be firmly established. Because the usefulness, safety, and feasibility of FOLFOX therapy has not been adequately demonstrated after resection of liver metastases in patients with CRC, investigators in Japan and other countries have criticized its indiscriminant use in patients after hepatectomy.

UFT (Taiho Pharmaceutical Company, Tokyo, Japan) is an oral 5-fluorouracil preparation combining tegafur and uracil in a molar ratio of 1:4. Tegafur is metabolized to 5-fluorouracil in the liver, and uracil competitively inhibits dihydropyrimidine dehydrogenase (DPD), the main metabolizing enzyme of 5-fluorouracil, thereby increasing serum concentrations of 5-fluorouracil and enhancing antitumor activity. The National Surgical Adjuvant Breast and Bowel Project (NSABP) C-06 trial demonstrated that UFT/LV therapy is noninferior to 5 -fluorouracil/LV therapy as postoperative adjuvant chemotherapy for stage II or III colon cancer, establishing UFT/LV as a standard therapy of stage III CRC in Japan (15). It was speculated that UFT/LV would be a candidate as a novel treatment strategy for CRLM.

For this reason, we focused on UFT/LV adjuvant therapy which was approved in 2003 and initiated a phase III clinical trial to compare the effectiveness and safety of postoperative adjuvant chemotherapy with UFT/LV with those of surgery alone in Japanese patients who underwent resection of liver metastases from colorectal cancer from 2004. This study is registered in the UMIN Clinical Trials Registry (registration ID number: UMIN: C000000013, http://www.umin.ac.jp/ ctr/index-j.htm). Although the primary endpoint (3-year relapse free survival (RFS)) is not found until 2014, we report the results of an interim analysis of the treatment completion rate, relative dose intensity, and safety of UFT/LV therapy. Because the safety and feasibility of adjuvant chemotherapy after hepatectomy remain unclear, reporting on safety in this study is expected to contribute to the optimal use of adjuvant chemotherapy after resection of liver metastases.

\section{Materials and Methods}

\subsection{Patients}

The trial was approved by the medical ethics committees of all participating centres and was conducted in accordance with the principles of the Declaration of Helsinki. Written informed consent was obtained from all patients before enrollment.

Eligible patients had to satisfy the following criteria: an age of 20 to younger than 80 years; a histopathologically confirmed diagnosis of liver metastasis from CRC; surgical resection of liver metastasis; macroscopically curative hepatectomy; initial treatment for liver metastasis or one previous resection of liver metastasis (either synchronous or metachronous); no extrahepatic lesions; no previous local or systemic chemotherapy or radiotherapy for liver metastasis; adequate organ functions at the start of treatment after surgery (white-cell count 4,000-12,000/ $\mu \mathrm{L}$, platelet count $\geq 100 \times 10^{3} / \mu \mathrm{L}$, hemoglobin level $\geq 9.0 \mathrm{~g} / \mathrm{dL}$, total bilirubin level $\leq 1.5 \mathrm{mg} / \mathrm{dL}$, aspartate aminotransferase and alanine aminotransferase levels $\leq$ $100 \mathrm{IU} / \mathrm{L}$, prothrombin activity $\geq 50 \%$, serum creatinine level $\leq 1.5 \mathrm{mg} / \mathrm{dL}$, blood urea nitrogen level $\leq 25 \mathrm{mg} / \mathrm{dL}$, total protein level $\geq 5.9 \mathrm{~g} / \mathrm{dL}$, albumin level $\geq 3.0 \mathrm{~g} / \mathrm{dL}$, C-reactive protein level $\leq 2.1 \mathrm{ng} / \mathrm{mL}$ ); and a performance status of 0 to 2 .

Patients were excluded if they had another active cancer, a clearly positive surgical margin at the time of hepatectomy, or serious postoperative complications. Pregnant or breast-feeding women were excluded. Patients with any of the following concurrent conditions were also excluded: receiving insulin treatment; poorly controlled diabetes mellitus or hypertension; a history of myocardial infarction within the past 6 months or unstable angina; liver cirrhosis; or interstitial pneumonia, pulmonary fibrosis, or pulmonary emphysema).

\subsection{Procedures}

Protocol treatment was started within 8 weeks after surgery. In the surgery alone group, patients were postoperatively followed up with further no treatment until metastasis or recurrence was confirmed. In the UFT/LV group, UFT ( $300 \mathrm{mg} / \mathrm{m}^{2} /$ day as tegafur) and LV (75 mg/day) were simultaneously given after meals 3 times per day for 28 days, followed by a 7-day rest. 
This was regarded as 1 course of treatment. This cycle was repeated until patients had received 5 courses $(25$ weeks) of UFT/LV therapy. The treatment criteria for UFT/LV therapy were as follows: white-cell count $\geq 4,000 / \mu \mathrm{L}$, platelet count $\geq 100 \times 10^{3} / \mu \mathrm{L}$, aspartate aminotransferase and alanine aminotransferase levels $<100 \mathrm{IU} / \mathrm{L}$, total bilirubin level $\leq 1.5 \mathrm{mg} / \mathrm{dL}$, and no grade 1 or higher nonhematologic toxicity, with the exception of constipation and hair loss. If the treatment criteria were not met because of adverse events at the scheduled time of starting a course of therapy, treatment was postponed until the criteria were satisfied. If the treatment criteria were not met during a course of therapy, the study treatment was discontinued and resumed when the criteria were met again. If the following criteria were met during a course of therapy, treatment with UFT was discontinued at the scheduled time of treatment resumption according to predesignated criteria: white-cell count $\leq 1,000 / \mu \mathrm{L}$ or platelet count $<$ $25 \times 10^{3} / \mu \mathrm{L}$, grade 3 or higher nonhematologic toxicity, or the criteria for the resumption of treatment were met from after 9 days to 15 days after discontinuing therapy. Once the dose of UFT was reduced, it was not increased again, even if toxicity resolved. The dose of LV was not changed. Protocol treatment with UFT/LV therapy was discontinued in the event of any of the following conditions: recurrence occurred; treatment could not be resumed for more than 15 days because of toxicity; the dose had to be reduced by more than one level because of toxicity; the patient requested withdrawal of the protocol treatment; death occurred during the protocol treatment; the protocol treatment was violated; the patient was found to be ineligible; or the physician in charge considered it difficult to continue the protocol treatment.

\subsection{Evaluation of safety}

Adverse events were monitored until 30 days after the final treatment and were evaluated according to the National Cancer Institute Common Terminology Criteria for Adverse Events, version 3.0. The worst grade of each adverse event was recorded.

\subsection{Statistical analysis}

The primary endpoint of the study was 3-year relapse-free survival. The sample size was planned approximately $20 \%$ for the surgery arm and $35 \%$ for the UFT/LV arm with power $75 \%$ at the 2 -sided $5 \%$ significance level, requiring 180 patients. Rates of relapse-free survival were estimated by the KaplanMeier method and compared by the logrank test. Secondary endpoints were overall survival, relapsefree period in the residual liver, and relapse-free period in other organs. The relative dose intensity (RDI) of UFT/LV therapy was calculated as follows: RDI = total administered dose divided by the total planned dose according to the study protocol $\times 100(\%)$.

\section{Results}

\subsection{Patients characteristics}

From January 2004 through December 2010 a total of 180 patients were enrolled at 11 hospitals. Ten patients were excluded, and the other 170 were included in safety analysis ( 88 in the surgery alone group and 82 in the UFT/LV group) (Figure 1). The reasons for exclusion were as follows: 2 patients assigned to the surgery alone group mistakenly received UFT/LV

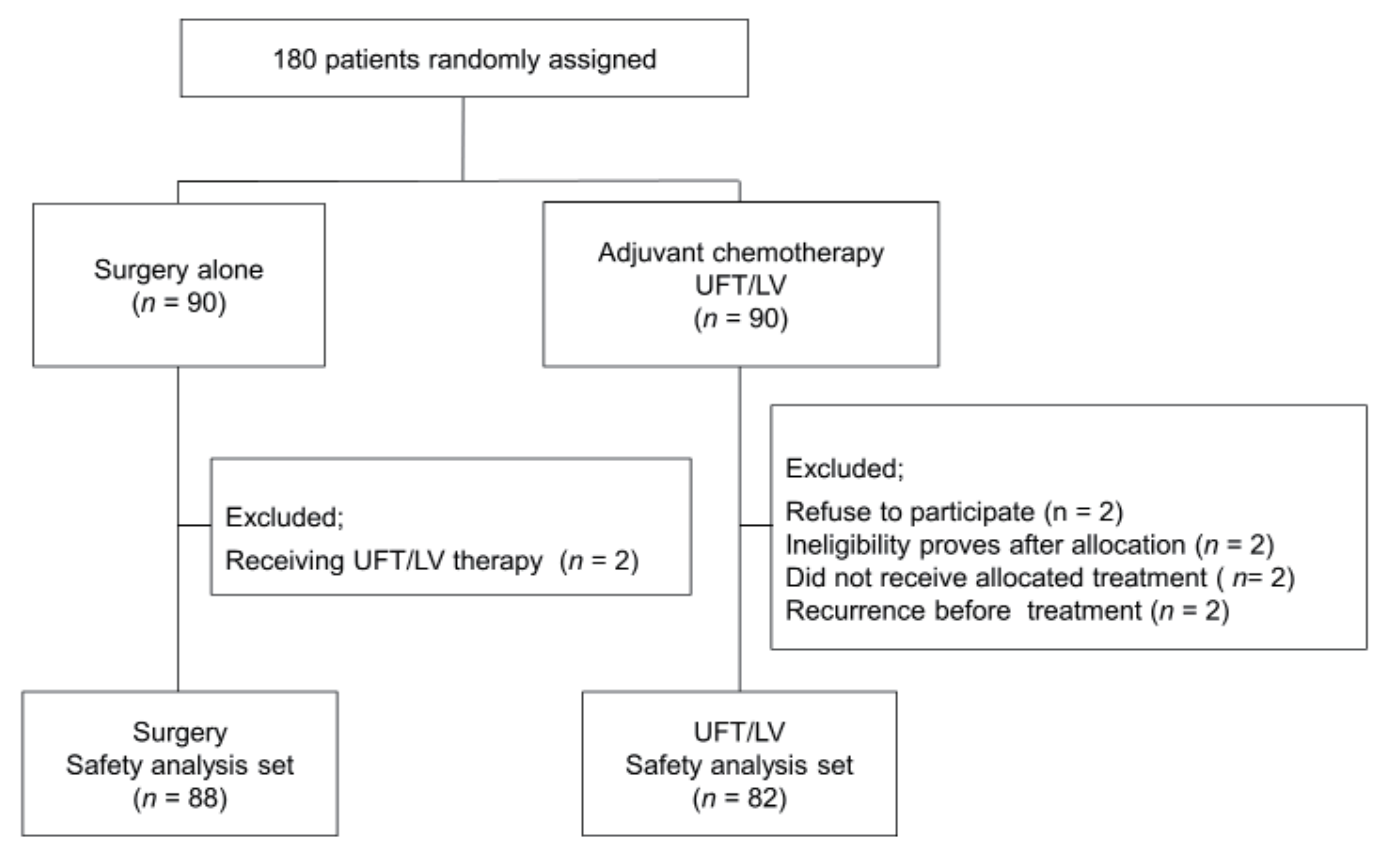

Figure 1. CONSORT diagram 
Table 1. Baseline characteristics

\begin{tabular}{lcc}
\hline Characteristics & $\begin{array}{c}\text { UFT/LV }(n=90) \\
n(\%)\end{array}$ & $\begin{array}{c}\text { Surgery alone }(n=90) \\
n(\%)\end{array}$ \\
\hline Sex & & \\
$\quad$ Male & $59(65.6)$ & $63(70.0)$ \\
$\quad$ Female & $31(34.4)$ & $27(30.0)$ \\
Age (years), mean (S.D.) & $62.2(8.5)$ & $64.5(9.2)$ \\
Location of primary tumor & & \\
$\quad$ Colon & $54(60.0)$ & $59(65.6)$ \\
$\quad$ Rectum & $36(40.0)$ & $31(34.4)$ \\
Tumor number & & \\
$\quad$ Single & $38(42.2)$ & $44(48.9)$ \\
$\quad$ Multiple & $52(57.8)$ & $46(51.1)$ \\
Size of largest tumor (mm) & & \\
$\quad$ 30 & $46(51.1)$ & $49(54.4)$ \\
$\quad 30<\leq 50$ & $23(25.6)$ & $23(25.6)$ \\
$\quad 50<$ & $21(23.3)$ & $18(20.0)$ \\
Timing of liver metastasis & & \\
$\quad$ Synchronous & $39(43.3)$ & $40(44.4)$ \\
$\quad$ Metachronous & $51(56.7)$ & $50(55.6)$ \\
Type of hepatectomy & & \\
$\quad$ Partial resection & $61(67.8)$ & $61(67.8)$ \\
$\quad$ Subsegmentectomy & $2(2.2)$ & $6(6.7)$ \\
$\quad$ Segmentectomy & $13(14.4)$ & $7(7.8)$ \\
$\quad$ Lobectomy & $14(15.6)$ & $16(17.7)$ \\
\hline
\end{tabular}

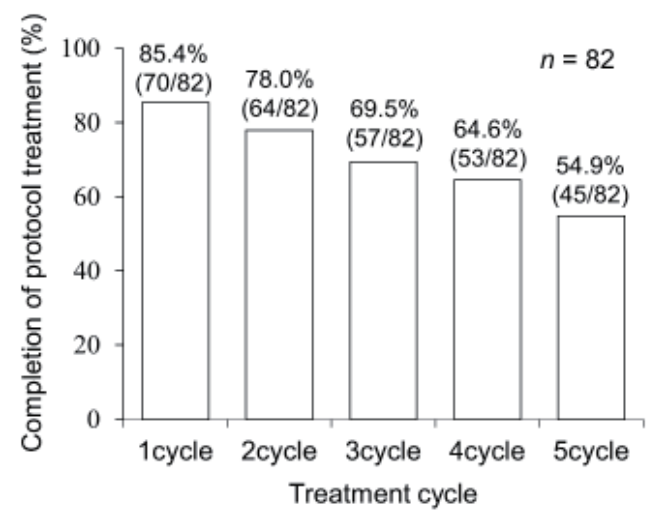

Figure 2. Completion of protocol treatment including those who had recurrence or discontinued treatment. therapy, and 8 patients assigned to the UFT/LV group did not receive the study drugs. Table 1 shows the baseline characteristics of all enrolled patients (Table 1).

\subsection{Treatment status}

Among the 82 patients who received UFT/LV therapy, 45 (54.9\%) completed the protocol treatment (5 courses). The proportion of patients according to the number of completed courses of protocol treatment was $85.4 \%$ (70 patients) for 1 course, $78.0 \%$ (64 patients) for 2 courses, $69.5 \%$ (57 patients) for 3 courses, and 64.6\% (53 patients) for 4 courses (Figure 2). The protocol treatment was discontinued in 37 patients. The main reasons for treatment withdrawal were adverse events in 26 patients (70.3\%), 19 of which discontinued because of the patient's or physician's discretion, recurrence in $8(21.6 \%)$ and unknown reasons in $3(8.1 \%)$. The most common cause of treatment withdrawal due to adverse events was grade 3 or 4 diarrhea, and treatment withdrawal at the patient's or physician's discretion were grade 1 or 2 mild adverse events (grade 2: diarrhea was common; grade 1: anorexia, stomatitis, diarrhea were common). The RDI of UFT/LV therapy was $70.8 \%$, with a median value of $90.0 \%$ (Figure 3).

\subsection{Safety}

Among the 82 patients in the UFT/LV group who were included in the safety analysis, $67(81.7 \%)$ had adverse events (all grades), and 10 (12.2\%) had grade 3 or 4 adverse events. Table 2 shows the adverse event profiles of the patients who were included in safety analysis. Grade 3 or 4 hematologic toxicity developing after UFT/LV therapy comprised decreased hemoglobin levels in 3 patients (3.7\%) and febrile neutropenia in $1(1.2 \%)$. Grade 3 or 4 nonhematologic toxicity

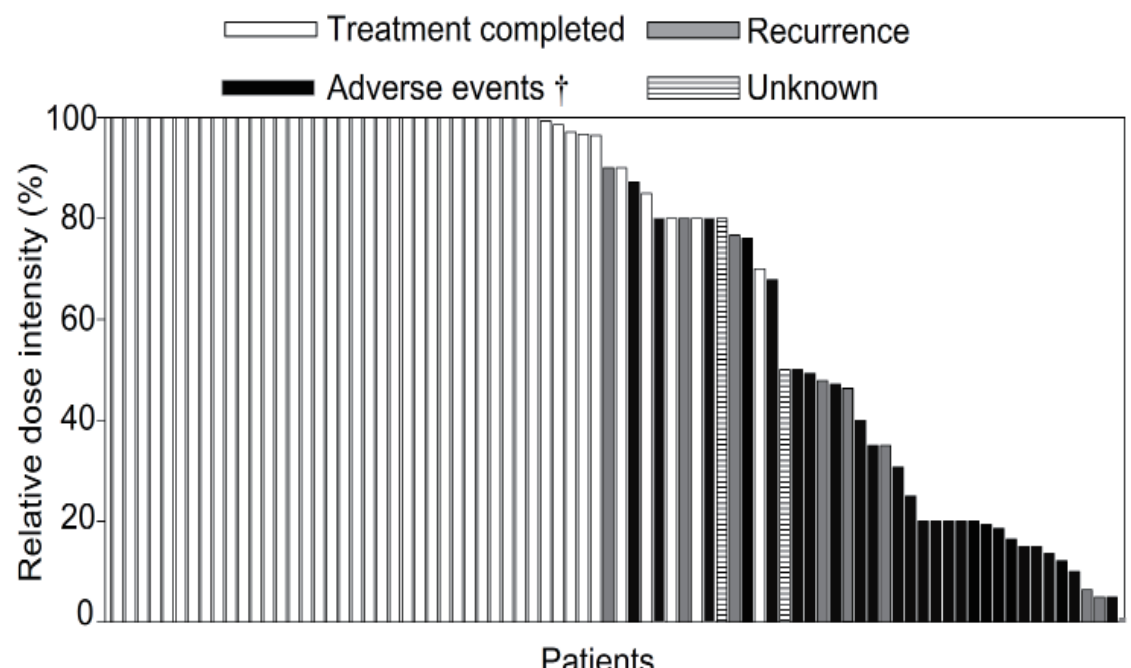

Figure 3. Relative dose intensity in the UFT/LV group including patients with recurrence. Each bar represents the percentage of relative dose intensity in each patient. The main reasons for treatment withdrawal were adverse events in $26(70.3 \%$, black bar), recurrence in $8\left(21.6 \%\right.$, gray bar) and unknown in $3\left(8.1 \%\right.$, gray bar with horizontal line). ${ }^{\dagger} 19$ patients discontinued treatment because of the patient's or physician's judgment. 
Table 2. Frequency of common toxic effects (worst grade)

\begin{tabular}{|c|c|c|c|c|c|c|c|c|}
\hline \multirow{3}{*}{ Adverse events } & \multicolumn{4}{|c|}{ UFT/LV $(n=82)$} & \multicolumn{4}{|c|}{ Surgery $(n=88)$} \\
\hline & \multicolumn{2}{|c|}{ Grade1, 2} & \multicolumn{2}{|c|}{ Grade3, 4} & \multicolumn{2}{|c|}{ Grade1, 2} & \multicolumn{2}{|c|}{ Grade 3,4} \\
\hline & $n$ & $(\%)$ & $n$ & $(\%)$ & $n$ & $(\%)$ & $n$ & $(\%)$ \\
\hline Leukocytes & 12 & 14.6 & 0 & 0 & 3 & 3.4 & 0 & 0 \\
\hline Platelets & 10 & 12.2 & 0 & 0 & 7 & 8.0 & 0 & 0 \\
\hline Haemoglobin & 13 & 15.9 & 3 & 3.7 & 7 & 8.0 & 1 & 1.1 \\
\hline Fibrile neutropenia & 0 & 0 & 1 & 1.2 & 0 & 0 & 0 & 0 \\
\hline AST & 13 & 15.9 & 2 & 2.4 & 4 & 4.5 & 0 & 0 \\
\hline ALT & 18 & 22.0 & 1 & 1.2 & 9 & 10.2 & 0 & 0 \\
\hline Total bilirubin & 22 & 26.8 & 1 & 1.2 & 5 & 5.7 & 0 & 0 \\
\hline ALP & 3 & 3.7 & 0 & 0 & 1 & 1.1 & 0 & 0 \\
\hline Diarrhea & 18 & 22.0 & 4 & 4.9 & 1 & 1.1 & 0 & 0 \\
\hline Anorexia & 21 & 25.6 & 2 & 2.4 & 0 & 0 & 0 & 0 \\
\hline Nausea & 9 & 11.0 & 2 & 2.4 & 1 & 1.1 & 0 & 0 \\
\hline Vomiting & 0 & 0 & 0 & 0 & 1 & 1.1 & 0 & 0 \\
\hline Stomatitis & 9 & 11.0 & 0 & 0 & 0 & 0 & 0 & 0 \\
\hline Fever & 4 & 4.9 & 0 & 0 & 2 & 2.3 & 0 & 0 \\
\hline Hand-foot skin reaction & 3 & 3.7 & 0 & 0 & 0 & 0 & 0 & 0 \\
\hline Hyperpigmentation & 1 & 1.2 & 0 & 0 & 0 & 0 & 0 & 0 \\
\hline Dysgeusia & 4 & 4.9 & 0 & 0 & 0 & 0 & 0 & 0 \\
\hline Neuropathy & 1 & 1.2 & 0 & 0 & 0 & 0 & 0 & 0 \\
\hline Fatigue & 5 & 6.1 & 0 & 0 & 0 & 0 & 0 & 0 \\
\hline
\end{tabular}

Abbreviations: AST, aspartate aminotransferase; ALT, alanine aminotransferase; ALP, alkaline phosphatase.

comprised elevated aspartate aminotransferase levels in 2 patients $(2.4 \%)$, elevated alanine aminotransferase levels in $1(1.2 \%)$, elevated bilirubin levels in $1(1.2 \%)$, diarrhea in $4(4.9 \%)$, anorexia in $2(2.4 \%)$, and nausea in $2(2.4 \%)$. There was no treatment-related death in the UFT/LV group.

\section{Discussion}

Patients undergoing curative resection of primary and metastatic liver tumors have been reported to achieve approximately $35 \%$ (17). But relapse is common after resection with two thirds of patients $(18,19)$. Adjuvant chemotherapy improves survival in patients with stage III CRC, but the role of adjuvant chemotherapy after resection of CRLM is still unknown. In this paper, we reported the safety and feasibility data from the multicenter phase III study of 180 patients with CRLM, who underwent UFT/LV or surgery alone. UFT/LV is one of the most widely used regimens and is recommended as a standard care for postoperative adjuvant chemotherapy for CRC in Japan.

In the NSABP C-06 trial, conducted in the United, $95.3 \%$ of the 774 patients who received UFT/LV therapy had adverse events (grade 3 or higher adverse events, 38.2\%) (15). In the ACTS-CC trial (Clinical Trials.gov: No. NCT00660894), a phase III controlled study designed to verify the noninferiority of S-1 to $\mathrm{UFT} / \mathrm{LV}$, a total of 1,535 patients have been enrolled, among whom 748 received UFT/LV therapy. Mochizuki et al. have reported on safety in the ACTS-CC trial (20). In the UFT/LV group, the incidence of adverse events was $73.7 \%$ for all grades and $14.4 \%$ for grades 3 or higher. The completion rate of UFT/LV therapy was
$73.4 \%$, and the RDI was $76.0 \%$. Recently presented data suggest outcome (21).

In the JCOG0205 trial (22), the 3-year diseasefree survival, the primary endpoint of the study, was $79.3 \%$ in the UFT/LV group and $77.8 \%$ in the 5 -fluorouracil/LV group (hazard ratio $=1.016,91.3 \%$ confidence interval, 0.838 to 1.232 , one-sided $p=$ 0.0236), demonstrating the non-inferiority of UFT/LV therapy to 5-fluorouracil/LV therapy. The completion rate of protocol treatment was $78 \%$ in both groups combined, indicating good treatment continuity. As for safety, the incidence of grade 3 or 4 increased alanine aminotransferase (ALT) and aspartate aminotransferase (AST) levels was higher in UFT/LV, whereas the incidences of diarrhea and anorexia were similar in the groups. The results of this study showed that the safety of UFT/LV for CRLM is similar when used as postoperative adjuvant chemotherapy in patients with stage III CRC.

The question of whether postoperative chemotherapy should be useful may be considered marginal. Portier et al. performed a controlled study (FFCD9002 trial) to compare surgery alone with 6 months of treatment with 5-fluorouracil/LV in patients who underwent curative resection of liver metastases. The 5-year disease free survival (DFS) was significantly better in the 5-fluorouracil/LV group, but there was no significant difference between the groups in overall survival. Although protocol treatment was completed in 54 $(66.7 \%)$ of 81 patients, 20 patients $(24.7 \%)$ in the 5 -fluorouracil/LV group had grade 3 or higher adverse events such as hematologic toxicity, stomatitis, nausea, and diarrhea. Twelve patients (14.8\%) experienced more than grade 3 to 4 toxicity (23). In the CPT- 
Table 3. Feasibility of different chemotherapeutic regimens in previous studies in patients with initially reserved liver metastases from colorectal cancer

\begin{tabular}{lclc}
\hline References & Number of patients & Randomised postoperative treatments & Complete treatment rate \\
\hline Portier G et al., 2006 (23) & 86 vs. 87 & Systemic FU/FA vs. surgery alone & $66.7 \%$ \\
Ychou M et al., 2009 (24) & 153 vs. 153 & Systemic FU/FA vs. FOLFIRI & $82 \%$ \\
& & & $75 \%$ \\
\hline Nordlinger B et al., 2008 (17) & Number of patients & Randomised perioperative treatments & Complete treatment rate \\
\hline
\end{tabular}

Abbreviations: FU, fluorouracil; FA, folinic acid; PeriOpCT, perioperative chemotherapy with FOLFOX4; preOp, preoperative chemotherapy; postop, postoperative chemotherapy. ${ }^{*} 115$ patients started postoperative chemotherapy, of whom $80(70 \%)$ received six cycles.

GMA-301 trial (24), which compared the usefulness of FOLFIRI (folinic acid, 5-fluorouracil, and irinotecan) therapy with that of 5 -fluorouracil/LV therapy, there was no statistically significant difference in the primary endpoint of disease-free survival between the groups. The incidence of grade 3 or 4 toxicity was $30 \%$ in the 5 -fluorouracil/LV group and $47 \%$ in the FOLFIRI group. In the FOLFIRI group, 22 patients (14\%) had grade 3 or 4 diarrhea, and $36(23 \%)$ had grade 3 or 4 neutropenia. Even in the 5-fluorouracil/LV control group, 11 patients $(7 \%)$ had grade 3 or higher diarrhea, and $10(7 \%)$ had neutropenia (Table 3). For these reasons, our results indicate that treatment UFT/LV after curative resection of liver metastases is associated with a lower incidence of grade 3 or 4 adverse events than conventional 5 -fluorouracil/LV therapy and is well tolerated.

Further improvements in treatment completion and adherence are required for postoperative adjuvant chemotherapy with UFT/LV to contribute to patient outcomes in clinical practice. In the FFCD trial and CPT-GMA-301 trial, the protocol treatment completion rate was $65 \%$ to $80 \%$. These results suggested that there is room for further improvement in the treatment completion rate of this regimen. In our study, the protocol treatment completion rate was $54.9 \%$ (45 of 82 patients, including those who discontinued treatment because of recurrence and $60.8 \%(45 / 74)$ when patients who discontinued treatment because of recurrence were excluded. Only 7 patients discontinued treatment because of grade 3 or higher adverse events that met the criteria for the withdrawal of protocol treatment. In about half of the patients who discontinued protocol treatment, therapy was withdrawn at the patient's request or physician's discretion because of grade 1 or 2 adverse events. Patient enrollment in our study was started in 2004. When the study began, UFT/LV therapy was not recognized to be a standard regimen for postoperative adjuvant chemotherapy in patients with colorectal cancer. Consequently, treatment was withdrawn in some patients because of relatively mild grade 2 or lower adverse events, leading to a treatment completion rate of only $55 \%$. Despite the debatable results, the RDI including patients who had recurrence or discontinued treatment was $70.8 \%$, a median value of $90.0 \%$, and without recurrence during chemotherapy was $73.2 \%$ and $97.9 \%$, respectively. As for mild adverse events, however, the compliance of individual patients can most likely be improved by obtaining fully informed consent before treatment and appropriate dose modification of drugs. UFT/LV therapy is thus considered a promising regimen for postoperative adjuvant chemotherapy in patients who undergo resection for liver metastases from colorectal cancer.

Recently, Nordlinger et al. reported the EORTC trial (40983) (17). In that study, secondary evaluations of eligible patients and those who underwent hepatectomy showed that the 3-year progression-free survival (PFS) significantly differed between the perioperative chemotherapy group and the surgery alone group. However, an intention-to-treat analysis revealed that the 3-year PFS did not differ significantly between the groups $(28.1 \% v s .35 .4 \%, p=0.058)$. The final results for the secondary endpoint of overall survival were presented at the 2012 annual meeting of American Society of Clinical Oncology (ASCO). The addition of perioperative chemotherapy to resection led to no significant improvement in long term survival (HR $0.87,0.66-1.14, p=0.303)$, but there was a mere $4 \%$ improvement in the FOLFOX4 arm after 5-years (25). Perioperative FOLFOX therapy is considered a highrisk chemotherapeutic regimen in terms of safety. In a previous study, the completion rate of preoperative FOLFOX therapy according to protocol was $84 \%$ (143/171). A total of 115 patients could receive postoperative adjuvant chemotherapy, but only 80 (44\%) were able to complete postoperative FOLFOX therapy (Table 3) (17). Grade 3 or 4 adverse events occurring during preoperative and postoperative therapy were leukopenia (preoperative chemotherapy $6 \% v s$. postoperative chemotherapy $12 \%$ ), neutropenia ( $18 \%$ vs. $35 \%)$, diarrhea ( $8 \%$ vs. $5 \%$ ), nausea ( $4 \%$ vs. $4 \%)$ and peripheral neuropathy ( $2 \% v s .10 \%)$. These results indicate that perioperative chemotherapeutic regimens are far from being safe (Table 4). Moreover, the incidence of postoperative complications was 
Table 4. Reported incidences of adverse events with other regimens

\begin{tabular}{|c|c|c|c|c|c|c|c|c|c|c|}
\hline \multirow{3}{*}{ Items } & \multicolumn{2}{|c|}{$\begin{array}{l}\text { Portier et al., } 2006 \\
\text { FFCD trial (23) }\end{array}$} & \multicolumn{4}{|c|}{$\begin{array}{c}\text { Ychou et al., } 2009 \\
\text { CPT-GMA-301 trial (24) }\end{array}$} & \multicolumn{4}{|c|}{$\begin{array}{l}\text { Nordlinger et al., } 2008 \\
\text { EORTC40983 trial (17) }\end{array}$} \\
\hline & \multirow{2}{*}{$\begin{array}{l}\text { FU/LA } \\
(n=81) \\
\text { Grade } 3 / 4 \\
\quad(\%)\end{array}$} & \multirow{2}{*}{$\begin{array}{l}\begin{array}{l}\text { Surgery } \\
\text { alone } \\
(n=85)\end{array} \\
\text { Grade 3/4 } \\
(\%)\end{array}$} & \multicolumn{2}{|c|}{$\begin{array}{l}\text { FU/LA } \\
(n=152)\end{array}$} & \multicolumn{2}{|c|}{$\begin{array}{l}\text { FOLFIRI } \\
(n=154)\end{array}$} & \multicolumn{2}{|c|}{$\begin{array}{l}\text { Preoperative } \\
\text { chemotherapy } \\
(n=171)\end{array}$} & \multicolumn{2}{|c|}{$\begin{array}{l}\text { Postoperative } \\
\text { chemotherapy } \\
\quad(n=115)\end{array}$} \\
\hline & & & $\begin{array}{l}\text { All grades } \\
(\%)\end{array}$ & $\begin{array}{l}\text { Grade } 3 / 4 \\
(\%)\end{array}$ & $\begin{array}{l}\text { All grades } \\
(\%)\end{array}$ & $\begin{array}{l}\text { Grade } 3 / 4 \\
(\%)\end{array}$ & $\begin{array}{l}\text { All grades } \\
(\%)\end{array}$ & $\begin{array}{l}\text { Grade } 3 / 4 \\
(\%)\end{array}$ & $\begin{array}{l}\text { All grades } \\
(\%)\end{array}$ & $\begin{array}{l}\text { Grade } 3 / 4 \\
(\%)\end{array}$ \\
\hline Leukocytes & ) & - & 7 & 1 & 18 & 3 & - & 6 & - & $12^{\dagger}$ \\
\hline Neutropenia & $\zeta 7.4 \% *$ & - & 16 & 7 & 41 & 23 & - & 18 & - & 35 \\
\hline Haemoglobin & & - & - & - & - & - & - & $1^{\dagger}$ & - & $1 \dagger$ \\
\hline Diarrhea & 8.6 & - & 51 & 7 & 60 & 14 & - & $8^{\dagger}$ & - & $5^{\dagger}$ \\
\hline Constipation & - & - & 15 & 0 & 24 & 1 & - & - & - & - \\
\hline Stomatitis & 7.4 & - & 26 & 0 & 21 & 1 & - & $7 \dagger$ & - & 0 \\
\hline Nausea & 7.4 & - & 59 & 3 & 75 & 3 & - & $4 \dagger$ & - & $4 \dagger$ \\
\hline Vomiting & - & - & 24 & 3 & 42 & 5 & - & 4 & - & $3 \dagger$ \\
\hline Anorexia & - & - & 12 & 0 & 20 & 1 & - & - & - & - \\
\hline Anemia & - & - & 12 & 0 & 12 & 0 & - & - & - & - \\
\hline Fatigue & - & - & 16 & 1 & 21 & 1 & - & - & - & - \\
\hline Neuropathy & 2.5 & - & - & - & - & - & - & $2 \dagger$ & - & $10^{\dagger}$ \\
\hline Dysgeusia & - & - & - & - & - & - & - & $2 \dagger$ & - & $4 \dagger$ \\
\hline Hand-foot skin syndrome & - & - & - & - & - & - & - & 0 & - & 1 \\
\hline
\end{tabular}

$\dagger$ No grade 4 reported. * Hematologic event was $7.4 \%$.

significantly higher in the chemotherapy group $(25 \%$ vs. $16 \%, p=0.04$ ).

In particular, liver disorders caused by irinotecanbased regimen (FOLFIRI) and oxaliplation-based regimen (FOLFOX) include fatty liver, steatohepatitis (yellow liver) (26-28), and sinusoidal dilation (blue liver) $(29,30)$. Despite these findings, the results of the EORTC 40983 trial led to the recognition of "preoperative/ postoperative chemotherapy plus surgery" as a standard therapy for resectable liver metastases in Europe (31). The National Comprehensive Cancer Network guidelines recommend multidisciplinary treatment combining hepatectomy and chemotherapy such as FOLFOX for the management of liver metastases (32). At present, the Japan Clinical Oncology Group (JCOG) is currently conducting a randomized phase II/III study (JCOG0603) comparing surgery alone with surgery plus mFOLFOX6 therapy after curative resection of liver metastasis from colorectal cancer (33).

UFT/LV do not require the placement of a central venous port or continuous intravenous infusion, thereby reducing system patients' stress associated with port placement, decreasing complications, and prolonging the interval between hospital visits. From the viewpoint of medical professionals, the use of oral anticancer agents reduces the time and effort required to set up infusion systems and is thus more convenient and economical.

Because patients have to receive oral medication on their own initiative, they should be instructed that it is essential to take medication as directed, and efforts should be made to show that oral anticancer agents have different adverse event profiles from those of injectable preparations. Even after the starting treatment, efforts to improve patient care by providing supportive therapy and instruction on drug administration management are required to improve adherence to treatment regimens and thereby promote the continuation of treatment while maintaining patients' quality of life. The development of adjuvant chemotherapy that prevents postoperative recurrence and substantially improves outcomes after resection of liver metastases in patients with colorectal cancer is an urgent task.

In conclusion, our results suggest that oral UFT/LV therapy is a therapeutically useful regimen. The final analysis of the data from our study is scheduled to be performed in 2014, and further detailed results are awaited.

\section{References}

1. Hughes KS, Simon R, Songhorabodi S, et al. Resection of the liver for colorectal carcinoma metastases: A multiinstitutional study of patterns of recurrence. Surgery. 1986; 100:278-284.

2. Nordlinger B, Quilichini MA, Parc R, Hannoun L, Delva E, Huguet C. Hepatic resection for colorectal liver metastases. Influence on survival of preoperative factors and surgery for recurrences in 80 patients. Ann Surg. 1987; 205:256-263.

3. Scheele J, Stangl R, Altendorf-Hofmann A. Hepatic metastases from colorectal carcinoma: Impact of surgical resection on the natural history. Br J Surg. 1990; 77:12411246.

4. Doci R, Gennari L, Bignami P, Montalto F, Morabito A, Bozzetti F. One hundred patients with hepatic metastases from colorectal cancer treated by resection: analysis of prognostic determinants. Br J Surg. 1990; 78:797-801.

5. Kokudo N, Tada K, Seki M, Ohta H, Azekura K, Ueno M, Matsubara T, Takahashi T, Nakajima T, Muto T. Anatomical major resection versus nonanatomical limited resection for liver metastases from colorectal carcinoma. 
Am J Surg. 2001; 181:153-159.

6. Minagawa M, Makuuchi M, Torzilli G, Takayama T, Kawasaki S, Kosuge T, Yamamoto J, Imamura H. Extension of the frontiers of surgical indications in the treatment of liver metastases from colorectal cancer: long-term results. Ann Surg. 2000; 231:487-499.

7. Weber SM, Jarnagin WR, DeMatteo RP, Blumgart LH, Fong Y. Survival after resection of multiple hepatic colorectal metastases. Ann Surg Oncol. 2000; 7:643-650.

8. Tomlinson JS, Jarnagin WR, DeMatteo RP, Fong Y, Kornprat P, Gonen M, Kemeny N, Brennan MF, Blumgart LH, D'Angelica M. Actual 10-year survival after resection of colorectal liver metastases defines cure. J Clin Oncol. 2007; 25:4575-4580.

9. Kokudo N, Seki M, Ohta H, Azekura K, Ueno M, Sato T, Moroguchi A, Matsubara T, Takahashi T, Nakajima T, Aiba K. Effects of systemic and regional chemotherapy after hepatic resection for colorectal metastases. Ann Surg Oncol. 1998; 5:706-712.

10. de Gramont A, Figer A, Seymour M, et al. Leucovorin and fluorouracil with or without oxaliplatin as first-line treatment in advanced colorectal cancer. J Clin Oncol. 2000; 18:2938-2947.

11. Goldberg RM, Sargent DJ, Morton RF, Fuchs CS, Ramanathan RK, Williamson SK, Findlay BP, Pitot HC, Alberts SR. A randomized controlled trial of fluorouracil plus leucovorin, irinotecan, and oxaliplatin combinations in patients with previously untreated metastatic colorectal cancer. J Clin Oncol. 2004; 22:23-30.

12. André T, Boni C, Mounedji-Boudiaf L, Navarro M, Tabernero J, Hickish T, Topham C, Zaninelli M, Clingan P, Bridgewater J, Tabah-Fisch I, de Gramont A. Oxaliplatin, fluorouracil, and leucovorin as adjuvant treatment for colon cancer. N Engl J Med. 2004; 350:2343-2351.

13. André $\mathrm{T}$, Boni $\mathrm{C}$, Navarro $\mathrm{M}$, Tabernero J, Hickish $\mathrm{T}$, Topham C, Bonetti A, Clingan P, Bridgewater J, Rivera F, de Gramont A. Improved overall survival with oxaliplatin, fluorouracil, and leucovorin as adjuvant treatment in stage II or III colon cancer in the MOSAIC trial. J Clin Onco. 2009; 27:3109-3116.

14. Kuebler JP, Wieand HS, O'Connell MJ, et al. Oxaliplatin combined with weekly bolus fluorouracil and leucovorin as surgical adjuvant chemotherapy for stage II and III colon cancer: results from NSABP C-07. J Clin Oncol. 2007; 25:2198-2204.

15. Lembersky BC, Wieand HS, Petrelli NJ, O'Connell MJ, Colangelo LH, Smith RE, Seay TE, Giguere JK, Marshall ME, Jacobs AD, Colman LK, Soran A, Yothers G, Wolmark N. Oral uracil and tegafur plus leucovorin compared with intravenous fluorouracil and leucovorin in stage II and III carcinoma of the colon: results from National Surgical Adjuvant Breast and Bowel Project Protocol C-06. J Clin Oncol. 2006; 24:2059-2064.

16. Kopec JA, Yothers G, Ganz PA, Land SR, Cecchini RS, Wieand HS, Lembersky BC, Wolmark N. Quality of life in operable colon cancer patients receiving oral compared with intravenous chemotherapy: Results from National Surgical Adjuvant Breast and Bowel Project Trial C-06. J Clin Oncol. 2007; 25:424-430.

17. Nordlinger B, Sorbye H, Glimelius B, et al. Perioperative chemotherapy with FOLFOX4 and surgery versus surgery alone for resectable liver metastases from colorectal cancer (EORTC Intergroup trial 40983): a randomized controlled trial. Lancet. 2007; 371:10071016.
18. Nordlinger B, Guiguet M, Vaillant JC, Balladur P, Boudjema K, Bachellier P, Jaeck D. Surgical resection of colorectal carcinoma metastases to the liver. A prognostic scoring system to improve case selection, based on 1568 patients. Association Française de Chirurgie. Cancer. 1996; 77:1254-1262.

19. Jones RP, Jackson R, Dunne DF, Malik HZ, Fenwick SW, Poston GJ, Ghaneh P. Systematic review and metaanalysis of follow-up after hepatectomy for colorectal liver metastases. Br J Surg. 2012; 99:477-486.

20. Mochizuki I, Takiuchi H, Ikejiri K, et al. Safety of UFT/ LV and S-1 as adjuvant therapy for stage III colon cancer in phase III trial: ACTS-CC trial. Br J Cancer. 2012; 106:1268-1273.

21. Nakamoto Y, Ishiguro M, Yoshida M, Ikejiri K, Mochizuki I, Mochizuki H, Kotake K, Kameoka S, Takahashi K, Watanabe T, Watanabe M, Boku N, Tomita N, Matsubara Y, Sugihara K, ACTS-CC Study Group. Noninferiority of S-1 to UFT/LV as adjuvant chemotherapy for stage III colon cancer: A randomized phase III trial (ACTS-CC). J Clin Oncol. 2013; 31 (suppl; abstr 3518).

22. Shimada Y, Hamaguchi T, Moriya Y, et al. Randomized phase III study of adjuvant chemotherapy with oral uracil and tegafur plus leucovorin versus intravenous fluorouracil and levofolinate in patients ( pts ) with stage III colon cancer ( CC ): Final results of Japan Clinical Oncology Group study ( JCOG0205). J Clin Oncol. 2012; 30 (suppl; abstr 3524).

23. Portier G, Elias D, Bouche O, Rougier P, Bosset JF, Saric J, Belghiti J, Piedbois P, Guimbaud R, Nordlinger B, Bugat R, Lazurthes F, Bedenne L. Multicenter randomized trial of adjuvant fluorouracil and folinic acid compared with surgery alone after resection of colorectal liver metastases: FFCD ACHBTH AURC 9002 trial. J Clin Oncol. 2006; 24:4976-4982.

24. Ychou M, Hohenberger W, Thezenas S, Navarro S, Maurel J, Bokemeyer C, Shacham-Shmueli E, Rivera F, Kwok-Keung Choi C, Santoro A. A randomized phase III study comparing adjuvant 5 -fluorouracil/folinic acid with FOLFIRI in patients following complete resection of liver metastases from colorectal cancer. Ann Oncol. 2009; 20:1964-1970.

25. Nordlinger B, Sorbye H, Glimelius B, et al. Perioperative FOLFOX4 chemotherapy and surgery versus surgery alone for resectable liver metastases from colorectal cancer (EORTC 40983): long-term results of a randomised, controlled, phase 3 trial. Lancet Oncol. 2013; 14:12081215.

26. Kooby DA, Fong Y, Suriawinata A, Gonen M, Allen PJ, Klimstra DS, DeMatteo RP, D'Angelica M, Blumgart LH, Jarnagin WR. Impact of steatosis on perioperative outcome following hepatic resection. J Gastrointest Surg. 2003; 7:1034-1044.

27. Vauthey JN, Pawlik TM, Ribero D, Wu TT, Zorzi D, Hoff PM, Xiong HQ, Eng C, Lauwers GY, Mino-Kenudson M, Risio M, Muratore A, Capussotti L, Curley SA, Abdalla EK. Chemotherapy regimen predicts steatohepatitis and an increase in 90-day mortality after surgery for hepatic colorectal metastases. J Clin Oncol. 2006; 24:2065-2072.

28. Fernandez FG, Ritter J, Goodwin JW, Linehan DC, Hawkins WG, Strasberg SM. Effect of steatohepatitis associated with irinotecan or oxaliplatin pretreatment on resectability of hepatic colorectal metastases. J Am Coll Surg. 2005; 200:845-853.

29. Aloia T, Sebagh M, Plasse M, Karam V, Lévi F, 
Giacchetti S, Azoulay D, Bismuth H, Castaing D, Adam R. Liver histology and surgical outcomes after preoperative chemotherapy with fluorouracil plus oxaliplatin in colorectal cancer liver metastases. J Clin Oncol. 2006; 24:4983-4990.

30. Rubbia-Brandt L, Audard V, Sartoretti P, Roth AD, Brezault C, Le Charpentier M, Dousset B, Morel P, Soubrane O, Chaussade S, Mentha G, Terris B. Severe hepatic sinusoidal obstruction associated with oxalilatinbased chemotherapy in patients with metastatic colorectal cancer. Ann Oncol. 2004; 15:460-466.

31. Nordlinger B, Van Cutsem E, Gruenberger T, Glimelius B, Poston G, Rougier P, Sobrero A, Ychou M. Combination of surgery and chemotherapy and the role of targeted agents in the treatment of patients with colorectal liver metastases: recommendations from an expert panel. Ann Oncol. 2009; 20:985-992.

32. NCCN. Colon cancer guidelines v3 2014. http://www. nccn.org/professionals/physician_gls/f_guidelines.asp (accessed February 11, 2014).

33. Kanemitsu Y, Kato T, Shimizu Y, Inada Y, Shimada Y, Nakamura K, Sato A, Moriya Y. A randomized phase II/III trial comparing hepatectomy followed by mFOLFOX6 with hepatectomy alone as treatment for liver metastasis from colorectal cancer: Japan Clinical Oncology Group Study JCOG0603. Jpn J Clin Oncol. 2009; 39:406-409.

(Received January 28, 2014; Revised February 24, 2014; Accepted February 26, 2014) 